\title{
The impact of orthotopic reconstruction on female sexuality and quality of life after radical cystectomy for non-malignant bladder conditions
}

\author{
Chiara Borghi ${ }^{1}$, Margherita Manservigi ${ }^{1}$, Elena Sofia Milandri ${ }^{1}$, Carmelo Ippolito ${ }^{3}$, \\ Pantaleo Greco ${ }^{1}$, Lucio Dell'Atti ${ }^{2}$ \\ ${ }^{1}$ Department of Surgical Sciences, Section of Obstetrics and Gynecology, Azienda Ospedaliero-Universitaria Sant'Anna, \\ University of Ferrara, Cona (Ferrara), Italy; \\ 2 Division of Urology, Department of Clinical, Special and Dental Sciences, University Hospital "Ospedali Riuniti" School \\ of Medicine, Marche Polytechnic University, Ancona, Italy; \\ ${ }^{3}$ Department of Surgical Sciences, Section of Urology, Azienda Ospedaliero-Universitaria Sant'Anna, University of Ferrara, \\ Cona (Ferrara), Italy.
}

\begin{abstract}
Summary Objective: To review the literature on the impact on female quality of life and sexual function of orthotopic reconstruction after radical cystectomy for non-malignant bladder conditions. Radical cystectomy is commonly required to treat malignant conditions but may also be considered for the treatment of non-malignant diseases. These heterogeneous group of disorders includes interstitial cystitis, painful bladder syndrome, neurogenic bladder, haemorrhagic/radiation cystitis, endometriosis and refractory genitourinary fistula. Treatment begins with non-invasive medical therapies but, in non-responder cases, a surgical solution should be considered. Such invasive techniques include urinary diversion and reconstructive procedures that have an impact on healthrelated quality of life, physical, social, and mental status. Materials and methods: This narrative review research was done using the PubMed database up until 2020, July. All papers referring to cystectomy for benign indication were considered. Results: In comparison to other reconstructive options, orthotopic neobladder allows the restoration of a normal self-image and consequently it is the most suitable procedure when a surgical reconstruction is necessary for non-malignant conditions. However, women can face many disorders that impact on everyday life, such as voiding dysfunction or sexual activity problems. Conclusions: Scant data is available about quality of life, sexual life and self-perception in women treated by cystectomy for benign conditions and most literature is dedicated to those indicators in cancer patients. More research is needed to understand the tolerability and the quality of life results of the female population affected by benign conditions undergoing this kind of surgical approach.
\end{abstract}

KEY WORDS: Orthotopic bladder; Cystectomy; Quality of life; Woman; Benign conditions; Non malignant conditions; Endometriosis; Interstitial cystitis; Neurological bladder; Sexuality; Urinary symptoms.

Submitted 25 January 2021; Accepted 23 April 2021

\section{INTRODUCTION}

Cystectomy is commonly required to treat malignant conditions such as urothelial carcinoma of the bladder Cystectomy with urinary diversion may also be considered for the treatment of non-malignant conditions in patients who have failed previous conservative therapies. These benign conditions represent a heterogeneous group of disorders including interstitial cystitis, painful bladder syndrome, neurogenic bladder, hemorrhagic/radiation cystitis, endometriosis and refractory genitourinary fistula.

Treatment begins with non invasive medical therapies but, in refractory cases, a surgical solution should be considered. After cystectomy, a urinary diversion and reconstructive procedures are needed. Over the past decades, orthotopic neobladder became a widely accepted technique for urinary diversion and the preferred method after removal of the bladder. Initially, it was limited just to men; women were considered ineligible for this procedure because urethra-sparing orthotopic substitution was thought to be associated with an increased risk of local recurrence and voiding dysfunction. With the improved understanding of the female sphincter mechanism, however, this approach has become technically accepted in selected cases (1).

An important issue in the decision-making process, prior to urinary diversion in females, is health related quality of life, including the effects of reconstruction of neobladder on physical, social and mental status.

Comparing the orthotopic neobladder to the other reconstructive options, this approach allows the restoration of a normal self-image and then it is the most suitable procedure when a surgical reconstruction is necessary for non-malignant conditions. However, women undergoing cystectomy for benign bladder conditions can face many disorders that can impact on everyday life, such as voiding dysfunction or sexual activity problems. These symptoms and complications can be temporary or permanent. Voiding often has a major impact on quality of life and an improved quality of life after urinary diversion fails to be realized when voiding dysfunction arises (2). Voiding dysfunction following the orthotopic neobladder can be divided into failure to store urine, during daytime, nighttime or both, and failure to empty, requiring intermittent self-catheterization (3).

This paper reviews the literature on the impact of ortho-

No conflict of interest declared. 
topic reconstruction on female quality of life and sexual function after radical cystectomy for non-malignant bladder conditions.

\section{Materials AND Methods}

Research was done using the PUBMED database up until 2021, January. All papers referring to cystectomy for benign indication were considered. A combination of Medical Subject Headings (MeSH) terms was used.

The keywords were: orthotopic bladder, cystectomy, quality of life, sexuality, woman, benign, endometriosis, interstitial cystitis, radiation cystitis and neurogenic bladder. All titles and abstracts published in English were evaluated.

Each article was evaluated according to the inclusion criteria: studies reporting any surgical intervention to treat benign bladder conditions, orthotopic neobladder for benign condition, quality of life after cystectomy and reconstructive surgery with particular attention to female population. All studies were considered, with the exception of those performed on animals, comments, letters, editorials and case reports. Due to the high heterogeneity regarding the indication for surgery, surgical approach, anesthesia procedures and complications, only a non-systematic review of literature and a critical synthesis of clinical experiences was performed.

\section{RESULTS AND DISCUSSION}

The initial search yielded a total of 47 articles. Forty articles were excluded after title/abstract screening for not meeting inclusion criteria. A total of seven articles strictly related to our issue were finally evaluated and reviewed by the authors. The selection of papers is reported in Figure 1. Other papers were included because of the topic relevance.

\section{Non-malignant indications for cystectomy}

Few benign diseases include cystectomy with urinary diversion among the treatment options. All of them have behavioral and medical first line therapies but, when patients are refractory to these less invasive ones, they can be considered for surgery.

These conditions are included in the present review's purpose and here shortly described.

- Interstitial Cystitis/Painful Bladder Syndrome (IC/PBS): a chronic debilitating inflammatory disease of the bladder that mainly affects women, most often in the fourth decade or later. It is defined as an unpleasant sensation characterized by suprapubic pain and lower urinary

Figure 1.

Flow chart for paper selection.

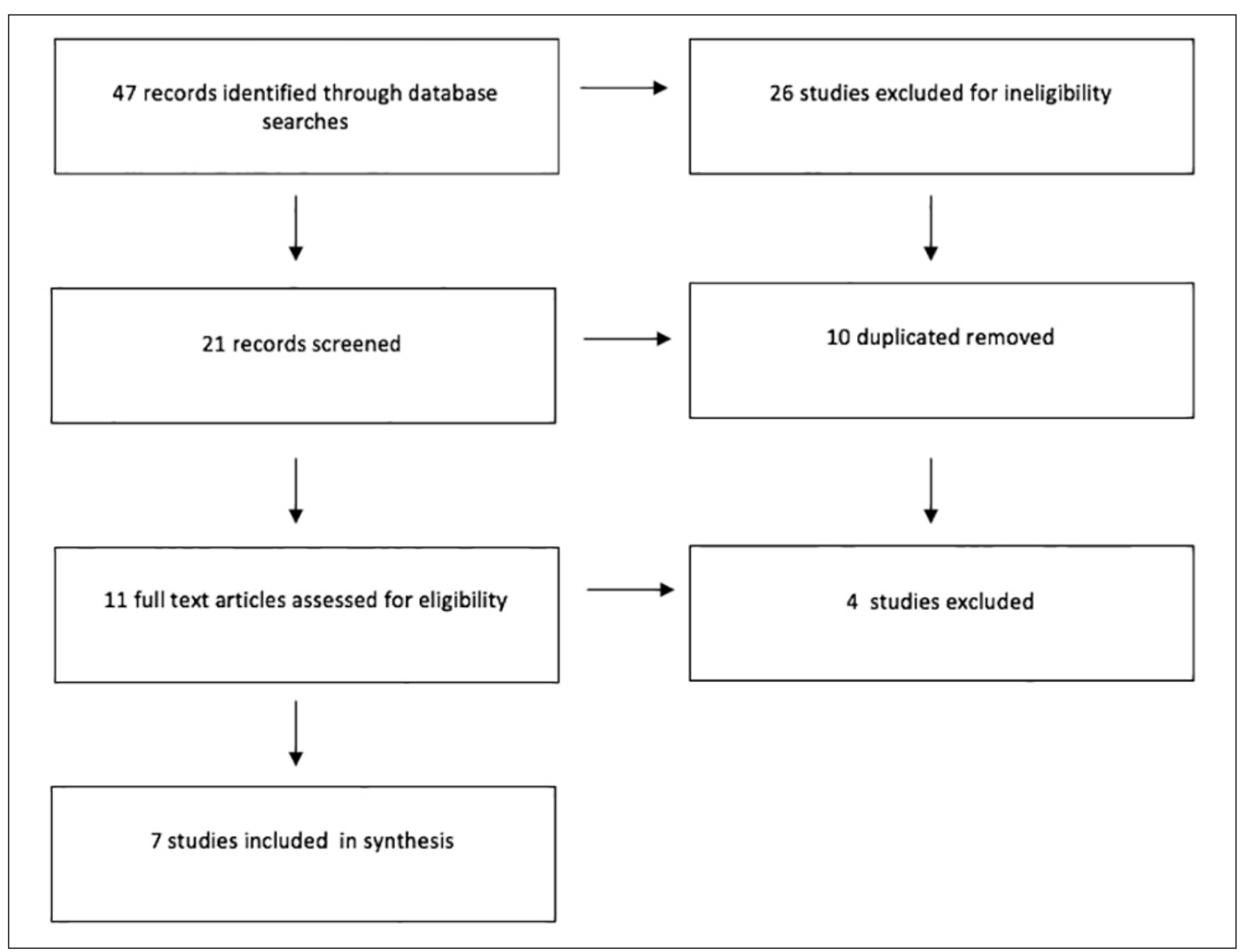


tract symptoms lasting more than six weeks, in absence of infection or other identifiable causes $(4,5)$.

- Neurogenic bladder: a condition that can be caused by congenital anomalies such as meningomyelocele and spina bifida or by acquired central nervous system diseases, such as spinal cord injury, multiple sclerosis, stroke, and parkinsonism (6). If left untreated, these pathological conditions can lead to a progression of urinary dysfunction. The diagnosis is based on clinical evaluation. Biological, radiological, and urodynamic investigations are indicated to plan the best management (6-8).

- Hemorrhagic cystitis is a condition characterized by hematuria and lower urinary tract symptoms. It can be acute or chronic, caused by trauma, infections, chemotherapy, and radiation (9-11). The diagnosis of hemorrhagic/radiation cystitis is based on past medical history, signs, symptoms, urine tests and cystoscopy (12).

- Genitourinary tuberculosis and schistosomiasis are infectious diseases endemic in developing countries that can cause severe urinary damage such as bladder fibrosis. About $30 \%$ of extra-pulmonary tuberculosis interest the genitourinary system. The most common symptoms of bladder tuberculosis are flank pain, dysuria, hematuria and frequency (13). The diagnosis is based on clinics, urine testing with sterile pyuria, histopathological examination and radiological exam (caverns of kidney) (14). All patients are treated with antibiotics, but in case of severe retracted bladder or serious reflux that damages the upper urinary tract, a surgical treatment could be necessary $(13,15)$.

In schistosomiasis, the host immune reaction to the bilharzial eggs results in healing by fibrosis and may progress to mucosal, submucosal, or muscular lesions, up to squamous cell carcinoma (16-18).

- Bladder endometriosis is defined as the infiltration of the endometrial tissue into the detrusor muscle. Characteristic symptoms include dysuria, frequency, hematuria, urgency and bladder pain, symptoms that may worsen during menstruation. Vaginal and physical examinations allow the identification of palpable nodules. Trans-vaginal ultrasonography should be regarded as a first line radiological test for the assessment of bladder endometriosis, while Magnetic Resonance is considered a second-line technique. Cystoscopy is used to assess the interior lining of the urethra and bladder (19). Conservative approaches can be used, such as the simple resection of endometriotic nodules, however, in selected cases, partial cystectomy is needed (20).

\section{Orthotopic neobladder - Surgical principles}

Orthotopic neobladder is one of the reconstructive urinary diversion procedures after cystectomy. Technique of orthotopic bladder aim to build a high capacity and low pressure reservoir and to connect it to the native urethra, proximally to the external striated sphincter.

The neobladder reservoir is made of de-tubularized gastric, ileum, ileocolonic or colonic segments. Maximal neobladder capacity depends on the choice and configuration of bowel segments harvested. In general, pressures are lower and capacities are greater in ileal segments. This procedure allows the patient to void volitionally through the urethra, restoring a more natural voiding pattern (21).

\section{Long-Term Quality of Life following cystectomy and urinary diversion with orthotopic bladder}

In the World Health Organization (WHO) concept of health and quality of life (QoL), the latter is defined as "an individual's perception of their position in life in the context of the culture and value systems in which they live and in relation to their goals, expectations and standards and concerns" (22). Health-related quality of life after surgery appears to be an important issue in the decision-making process prior to urinary diversion. Although disease or anatomic considerations may limit neobladder use in some patients, orthotopic bladder is commonly suitable to many of those undergoing cystectomy.

This procedure is associated with significant changes in urinary and sexual function, relationships, and psychosocial habits (23).

All these changes impact patients' perceived quality of life. Several groups analyzed QoL after radical cystectomy and reconstruction of neobladder for malignant conditions, comparing it with other types of urinary diversion, to clarify the effects on physical, social, and mental status. Some proponents of continent orthotopic diversion have used general quality of life instruments (i.e. 36-Item Short Form SF36) (24) to study patient outcomes and have cited incontinence bother, sexual function, and social comfort as possible reasons to use neobladder diversion $(25,26)$. Most investigators, however, have been unable to demonstrate significant differences in quality of life parameters among diversion groups.

For example, Hobisch at al. compared subjective morbidity of ileal neobladder versus ileal conduit urinary diversion to elucidate its influence on quality of life. Using the European Organization for Research and Treatment of Cancer Quality of Life Core Questionnaire (EORTC-QLQC30 (27) they found that patients with a neobladder needed a shorter period of rehabilitation, $92.8 \%$ of them did not feel handicapped at all and $87 \%$ had no feelings of sickness or illness. They also demonstrated that urinary leakage, odor, and wet clothing were much more common in patients with the stomal appliance of conduit than in the continent reservoir and neobladder patients (28). On the other hand, Hedgepeth at al. studied the body image and quality of life, evaluating urinary, sexual and bowel domains, and they did not find any difference between patients undergoing urostomy and those who had neobladder reconstruction (29).

There is a paucity of outcome data regarding quality of life after urinary diversion with orthotopic bladder for benign conditions in literature. In a study about bladder substitution by ileal neobladder in women with IC/PBS, authors found that all patients presented good treatment outcomes and quality of life by using SF36 questionnaire. They showed significant improvement of both physical and mental health (30). Moreover, the main factors affecting General Health QoL (GH-QoL) after neobladder substitution were voiding dysfunctions. In fact, patients with daytime incontinence or requiring clean intermittent catheterization (CIC), had lower SF36 scores than those without these symptoms $(2,30)$.

However, Cody and colleagues, in a Cochrane collaboration review, analyzed the different types of urinary diversion and the review did not find any significant evidence 
as to the superiority of continent vs incontinent diversion after cystectomy for malignant as well as non-malignant indications (31).

\section{Voiding dysfunctions in the orthotopic neobladder}

Voiding dysfunctions following orthotopic neobladder can be divided into urinary incontinence during the daytime, night-time or both, or the inability to empty the bladder ("hyper-continence") requiring CIC (32). Voiding disorders are frequent findings in female patients after orthotopic neobladder and can arise both from physiological and anatomical defects. Risk factors for the development of the daytime urinary incontinence include advanced age, inadequate storage reservoir and alteration of the sphincteric mechanism (3). Nerve sparing cystectomy is another factor believed to provide early continence in female patients, along with the preservation of the trigone with no damage to the autonomic nervous system. However, with the preservation of the trigone, a significant portion of patients develop urinary retention, which worsens over time. The highest rates of urinary retentionists are found where nerve-sparing technique is performed $(30,33)$. Another common effect of neobladder is night-time incontinence. It results from the overdistension of the neobladder and lack of voiding sensation. The patient fails to wake up during the night to empty the bladder, allowing excessive urinary volume to overcome the urethral closure mechanism.

The use of cystectomy with a neobladder for treatment of patients with bladder carcinoma has been reported with long term follow up, demonstrating that this operation is safe for female patients (34). However, care must be taken at the time of surgery to prevent future voiding problems. In fact, the post-operative reduction in terms of QoL is substantial when voiding dysfunctions occur following the neobladder. Patients with daytime incontinence or requiring CIC had lower SF36 scores than those without incontinence or CIC. However, General healthQuality of Life (GH-QoL) score in patients with enuresis was the same as that without enuresis, regardless of the degree of enuresis and urination QoL was not as high as surgeons expected (2). On the other hand, it was found that, even if the patient is incontinent and requires 5 to 6 sanitary pads daily, neobladder was preferable to maintain body image (1).

\section{Sexual function following cystectomy and urinary diversion with orthotopic bladder}

During radical cystectomy in women, the neurovascular bundles, placed on the lateral walls of the vagina, are usually removed or damaged by removal of the bladder, urethra, and anterior vaginal wall. Therefore, anterior exenteration may result in diminished ability or inability to achieve orgasm, decreased lubrication, decreased sexual desire and dyspareunia (35). Sexual dysfunction is a prevalent problem after female radical cystectomy and many of bladder cancer specific questionnaires, such as the Bladder Cancer Index (BCI), Vanderbilt Cystectomy Index (VCI), Functional Assessment of Cancer Therapy Bladder (FACT-B) and EORTC-QOL-B20, include sexual function and dysfunction items. For women, the most commonly used questionnaire is the Female Sexual Function Index (FSFI), that analyzes specific domains including the degree of vaginal lubrication, ability to achieve orgasm, degree of pain during intercourse, overall sexual desire and interest, and overall sexual satisfaction. Craig et al. conducted a clinical study to address sexual dysfunction in a subset of sexually active women undergoing radical cystectomy (RC) using a modified Index of Female Sexual Function questionnaire and strat-

\section{Table 1}

Studies about orthotopic neobladder for benign conditions, extraction of women population.

\begin{tabular}{|c|c|c|c|c|c|c|c|c|}
\hline $\begin{array}{l}\text { Authors, } \\
\text { year }\end{array}$ & $\begin{array}{l}\text { Type } \\
\text { of study }\end{array}$ & Disease & $\begin{array}{c}\text { Number } \\
\text { of benign cases }\end{array}$ & $\begin{array}{l}\text { Type of orthotopic } \\
\text { neobladder } \\
\text { after RC or SC }\end{array}$ & $\begin{array}{l}\text { Mean age } \\
\text { (years) }\end{array}$ & $\begin{array}{l}\text { QoL } \\
\text { (evaluated yes/no - } \\
\text { instrument if yes) }\end{array}$ & $\begin{array}{c}\text { LUTS } \\
\text { (evaluated } \\
\text { yes/no) }\end{array}$ & $\begin{array}{l}\text { Sexual function } \\
\text { (evaluated } \\
\text { yes/no) }\end{array}$ \\
\hline Gross et al., 2015 (37) & Observational & Post-radiation cystitis & 4 & RC with ileal orthotopic neobladder & 61.7 & No & Yes & No \\
\hline Cohn et al., 2014 (38) & Observational & $\begin{array}{l}\text { Infection, fistula, } \\
\text { bleeding, neurogenic } \\
\text { bladder or pain }\end{array}$ & $\begin{array}{c}2 \\
\text { (not clear if } \\
\text { men or women) }\end{array}$ & RC with ileal orthotopic neobladder & 57.8 & No & Yes & No \\
\hline Gobeaux et al., 2011 (39) & Observational & $\begin{array}{l}\text { Neurogenic detrusor } \\
\text { overactivity }\end{array}$ & 31 & $\begin{array}{c}\text { SC with ileal orthotopic } \\
\text { neobladder (modified Hautmann) }\end{array}$ & 34.7 & $\begin{array}{c}\text { Yes } \\
\text { (voiding diary, radiological, } \\
\text { laboratory, endoscopic } \\
\text { and urodynamic assessment) }\end{array}$ & Yes & No \\
\hline Takenaka et al., 2010 (2) & Observational & Contracted bladder & $\begin{array}{c}1 \\
\text { (not clear if } \\
\text { man or woman) }\end{array}$ & $\begin{array}{l}\text { RC with ileum or colon } \\
\text { as neobladder reservoirs }\end{array}$ & 62 & $\begin{array}{c}\text { Yes } \\
\text { (SF-36 questionnaire) }\end{array}$ & Yes & $\begin{array}{c}\text { No } \\
\text { (only in male) }\end{array}$ \\
\hline Kochakarn et al., 2007 (30) & Observational & $\begin{array}{l}\text { Interstitial cystitis } \\
\text { neobladder } \\
\text { (modified Sturder) }\end{array}$ & 35 & $\begin{array}{l}\text { RC with ileal orthotopic } \\
\text { (SF-36 questionnaire) }\end{array}$ & 45.9 & Yes & Yes & Yes \\
\hline Studer et al., 2005 (40) & Observational & Not specified & $\begin{array}{c}17 \\
\text { (not clear if } \\
\text { men or women) }\end{array}$ & RC with ileal orthotopic neobladder & 65 & No & Yes & $\begin{array}{c}\text { No } \\
\text { (only in male) }\end{array}$ \\
\hline Shimogaki et al., 1999 (1) & Observational & Contracted bladder & 2 & SC with ileal orthotopic neobladder & 45.5 & $\begin{array}{c}\text { Yes } \\
\text { (questionnaire about } \\
\text { voiding status, } \\
\text { continence and } \mathrm{QoL} \text { ) }\end{array}$ & Yes & No \\
\hline
\end{tabular}


ifying the sexual response by the type of urinary diversion to determine whether vaginal sparing (as performed in the orthotopic diversion) influenced the sexual response. No statistically significant difference was found between the Indiana external diversion stoma and the Studer orthotopic in women after RC (35).

When performed for benign conditions, cystectomy frequently does not need anterior pelvic exenteration. Nevertheless, sexual dysfunction may be related to neural injury and changes in pelvic anatomy. Other factors that may impact sexual satisfaction and libido include body alterations associated with urinary diversion, resulting in changes in body images, emotional and psychological responses of both patients and their partners, and the age-related changes in libido and sexual interest. Patients with orthotopic neobladder do not undergo the same degree of body alteration as others urinary diversion techniques, but the poor urine control and incontinence can be significant stressors that may limit interest in sexual activity (36). Moreover, preservation of normal sexual function in women has not been the main goal in most studies of neobladders in women.

In conclusion, orthotopic neobladder technique, used for the treatment of benign diseases, allows the improvement of both physical and mental health. Urinary symptoms remain the most frequently reported problems affecting general health and sexual function in operated women.

\section{Sexual function and quality of life in women after radical cystectomy for non-malignant bladder conditions} In Table 1 we listed the few articles taking into account the population of interest. Gross et al. in 2015 evaluated how hysterectomy and nerve sparing affected functional outcomes after ileal orthotopic bladder substitution in 73 women. Four of them underwent cystectomy for postradiation cystitis, the other 69 for invasive urothelial cancer. The results showed a strong correlation between postoperative urinary incontinence and pre- or perioperative hysterectomy.

These findings suggest that voiding disorders are related to the damage of autonomic nerves, which run along the lateral aspect of cervix uteri (37).

Cohn et al. (2014) investigated perioperative outcomes after cystectomy and urinary diversion for the treatment of refractory benign urological diseases, like infection, fistula, bleeding, incontinence, neurogenic bladder or pain. The study group included 8 males and 18 females; 22 patients underwent ileal conduit, 2 underwent ileal neobladder and 2 underwent Indiana pouch diversion. The authors demonstrated that cystectomy and urinary diversion resulted in resolution of urological symptoms in $73 \%$ of patients but the procedure was morbid for many, in fact $73 \%$ of patients experienced a complication within 30 days of surgery (urinary tract infection, abscess, urine leak etc) (38).

Gobeaux et al. (2011) evaluated continence status, urodynamic changes and long-term sequelae of Hautmann pouch following supratrigonal cystectomy in a population of 61 patients, 30 males and 31 females, with neurogenic detrusor overactivity. They found that this surgical technique achieved complete continence in $75 \%$ of cases, reduced rates of infection and dependency on pharmaco- logical agents. All these factors contributed to the improvement of QoL (39).

Takenaka et al. (2010) analyzed the general health QoL, urinary QoL, and sexual QoL in 78 males and 8 females five years after orthotopic neobladder substitution. Most of the population had bladder cancer or malignancies of other organs that had invaded the bladder. Only one patient, whose gender isn't specified, had a benign pathology. The authors found that although the general health QoL was generally well maintained, the presence of intermittent CIC or daytime incontinence impaired the QoL. Regarding sexual satisfaction and female sexual function, authors didn't examine these items (2).

Kochakarn et al. in 2007 reported their experience with cystectomy and ileal neobladder in 35 female patients with interstitial cystitis, in particular they assessed general QoL, voiding disorders and sexual life. They showed that QoL improved in both physical and mental health components; after 6 months diurnal and nocturnal continence were achieved in $100 \%$ of patients, spontaneous voiding was noted in 33 cases, the other 2 cases voided spontaneously with residual urine and used intermittent catheterization. Among 30 cases of sexually active patients, 12 had mild degree of dyspareunia during the first year, no patient had problems in sexuality after 1 year (30).

Studer et al. in 2005 presented long-term results of a large series of patients after ileal orthotopic bladder substitution. The study considered 482 patients including 40 women; cystectomy was performed for cancer in 465 cases and for other reasons in only 17 cases (not clear if males or females). The authors demonstrated that the bladder capacity was increased rapidly after surgery, daytime and nighttime frequency decreased during the first year then remained unchanged for 5 years and urinary infections occurred in $10 \%$ of patients, in association with residual urine. The sexual function was considered only in men (40).

Shimogaki et al. (1999) investigated the long-term outcome of orthotopic neobladders in 8 women, 6 of whom were treated for invasive bladder cancer and 2 for contracted bladder. In particular, they focused on QoL and voiding dysfunction. They found that patients' satisfaction was excellent, daytime and nighttime continence was achieved in $88 \%$ of the patients, although half of the women required intermittent catheterization (1).

In cases of failure of all conservative treatments, cystectomy with enterocystoplasty is used in many institutions for treating IC/PBS. At the beginning of bladder reconstruction in female patients, supratrigonal cystectomy has been used to avoid urinary incontinence. However, even leaving only vestigial bladder muscle, persistent painful bladder was still present. Webster et al. reported complete painful relief after additional removal of the trigone in patients submitted to supratrigonal cystectomy and enterocystoplasty for treating IC/PBS (41-43). In fact, urinary incontinence after a neobladder operation depends on creating adequate storage reservoir and preserving the sphincteric mechanism. Sectioning the urethra below bladder neck in female patients can maintain the continence mechanism with better emptying than in the case of bladder neck preservation (44). Nerve sparing cystec- 
tomy is another factor believed to provide early continence in women. Sparing of autonomic nerve fibers provide beneath the urethra was found to provide early urinary control in derived patients. Keeping endopelvic fascia intact not only preserves the nerve but also keeps urethra-pelvic ligament, enhancing urinary control (45).

\section{Conclusions}

Orthotopic neobladder is a complex surgical technique used for reconstruction of lower urinary tract after cystectomy. Such procedure is used for benign conditions only in cases of lack of response to conservative treatments. It allows preservation of normal body perception but, on the other hand, it is related to several possible adverse conditions and complications. Scant data is available about quality of daily life, sexual life and self-perception in women treated for benign conditions and most literature is dedicated to QoL indicators in cancer patients (46).

More research is still needed to better understand the tolerability and the Quality of Life results in the female population affected by benign conditions undergoing this kind of surgical approach.

\section{REFERENCES}

1. Shimogaki H, Okada H, Fujisawa M, et al. Long-term experience with orthotopic reconstruction of the lower urinary tract in women. J Urol. 1999; 161:573-577.

2. Takenaka A, Hara I, Soga H, et al. Assessment of long-term quality of life in patients with orthotopic neobladder followed for more than 5 years. Int Urol Nephrol. 2011; 43:749-754.

3. Steers WD. Voiding dysfunction in the orthotopic neobladder. World J Urol. 2000; 18:324-329.

4. Hanno P, Dmochowski R. Status of international consensus on interstitial cystitis/bladder pain syndrome/painful bladder syndrome: 2008 snapshot. Neurourol Urodyn. 2009; 28:274-286.

5. Hanno PM, Burks DA, Clemens JQ, et al. AUA guideline for the diagnosis and treatment of interstitial cystitis/bladder pain syndrome. J Urol. 2011; 185:2162-2170.

6. Dorsher PT, McIntosh PM. Neurogenic bladder. Adv Urol. 2012; 2012:816274.

7. Amarenco G, Sheikh Ismaël S, Chesnel C, et al. Diagnosis and clinical evaluation of neurogenic bladder. Eur J Phys Rehabil Med. 2017; 53:975-980.

8. Westney OL. The neurogenic bladder and incontinent urinary diversion. Urol Clin North Am. 2010; 37:581-592.

9. Numazaki Y, Kumasaka T, Yano N, et al. Further study on acute hemorrhagic cystitis due to adenovirus type 11. N Engl J Med. 1973; 289:344-347.

10. Dropulic LK, Jones RJ. Polyomavirus BK infection in blood and marrow transplant recipients. Bone Marrow Transplant. 2008; 41:11-18.

11. Stillwell TJ, Benson RC. Cyclophosphamide induced hemorrhagic cystitis: A review of 100 patients. Cancer 1988; 61:451-457.

12. Okaneya T, Kontani K, Komiyama I, Takezaki T. Severe cyclophosphamide-induced hemorrhagic cystitis successfully treated by total cystectomy with ileal neobladder substitution: A case report. J Urol. 1993; 150:1909-1910.
13. Gokce G, Kilicarslan H, Ayan S, et al. Genitourinary tuberculosis: A review of 174 cases. Scand J Infect Dis. 2002; 34:338-340.

14. Kulchavenya E. Best practice in the diagnosis and management of urogenital tuberculosis. Ther Adv Urol. 2013; 5:143-151.

15. Carl P, Stark L. Indications for surgical management of genitourinary tuberculosis. World J Surg. 1997; 21:505-510.

16. Khalaf I, Shokeir A, Shalaby M. Urologic complications of genitourinary schistosomiasis. World J Urol. 2012; 30:31-38.

17. Gray DJ, Ross AG, Li YS, McManus DP. Diagnosis and management of schistosomiasis. Bmj. 2011; 342:1-12.

18. Ghoneim MA, Shoukry I. The use of ileum for correction of advanced or complicated bilharzial lesions of the urinary tract. Int Urol Nephrol. 1972; 4:25-33.

19. Leone Roberti Maggiore U, Ferrero S, Candiani M, et al. Bladder endometriosis: a systematic review of pathogenesis, diagnosis, treatment, impact on fertility, and risk of malignant transformation. Eur Urol. 2017; 71:790-807.

20. Nezhat CH, Malik S, Osias J, et al. Laparoscopic management of 15 patients with infiltrating endometriosis of the bladder and a case of primary intravesical endometrioid adenosarcoma. Fertil Steril; 2002; 78:872-875

21. Gschwend JE. Bladder substitution. Curr Opin Urol. 2003; 13:477-482.

22. Measuring health-A review of Quality of Life measurement scales. Accessed July 31, 2020. https://www.ncbi.nlm.nih.gov/pmc/ articles/PMC1059623/

23. Mânsson A, Mânsson W. When the bladder is gone: Quality of life following different types of urinary diversion. World J Urol. 1999; 17:211-218.

24. Stansfeld SA, Roberts R, Foot SP. Assessing the validity of the SF36 general health survey. Qual Life Res. 1997; 6:217-224.

25. Bjerre BD, Johansen C, Steven K. Health-related quality of life after cystectomy: bladder substitution compared with ileal conduit diversion. A questionnaire survey. Br J Urol. 1995; 75:200-205.

26. Boyd SD, Feinberg SM, Skinner DG, et al. Quality of life survey of urinary diversion patients: comparison of ileal conduits versus continent Kock ileal reservoirs. J Urol. 1987; 138:1386-1389.

27. Aaronson NK, Ahmedzai S, Bergman B, et al. The European organization for research and $t$ reatment of cancer QLQ-C30: A quality-of-life instrument for use in international clinical trials in oncology. J Natl Cancer Inst. 1993; 85:365-376.

28. Hobisch A, Tosun K, Kinzl J, et al. Quality of life after cystectomy and orthotopic neobladder versus ileal conduit urinary diversion. World J Urol. 2000; 18:338-344.

29. Hedgepeth RC, Gilbert SM, He C, et al. Body image and bladder cancer specific quality of life in patients with ileal conduit and neobladder urinary diversions. Urology. 2010; 76:671-675.

30. Kochakarn W, Lertsithichai P, Pummangura W. Bladder substitution by ileal neobladder for women with interstitial cystitis. Int Braz J Urol. 2007; 33:486-492.

31. Cody JD, Nabi G, Dublin N, et al. Urinary diversion and bladder reconstruction/replacement using intestinal segments for intractable incontinence or following cystectomy. Cochrane Database Syst Rev. 2012; 2012:CD003306

32. Park JM, Montie JE. Mechanisms of incontinence and retention after orthotopic neobladder diversion. Urology. 1998; 51:601-609. 
33. Chiang PH, Huang YS, Wu WJ, et al. Orthotopic bladder substitution in women using the ileal neobladder. J Formos Med Assoc. 2000; 99:348-351.

34. Hautmann RE, Volkmer BG, Schumacher MC, et al. Long-term results of standard procedures in urology: The ileal neobladder. World J Urol. 2006; 24:305-314.

35. Zippe $C D$, Raina $R$, Shah $A D$, et al. Female sexual dysfunction after radical cystectomy: A new outcome measure. Urology. 2004; 63:1153-1157.

36. Modh RA, Mulhall JP, Gilbert SM. Sexual dysfunction after cystectomy and urinary diversion. Nat Rev Urol. 2014; 11:445-453.

37. Gross T, Meierhans Ruf SD, Meissner C, et al. Orthotopic ileal bladder substitution in women: Factors influencing urinary incontinence and hypercontinence. Eur Urol. 2015; 68:664-671.

38. Cohn JA, Large MC, Richards KA, et al. Cystectomy and urinary diversion as management of treatment-refractory benign disease: The impact of preoperative urological conditions on perioperative outcomes. Int J Urol. 2014; 21:382-386.

39. Gobeaux N, Yates DR, Denys P, et al. Supratrigonal cystectomy with Hautmann pouch as treatment for neurogenic bladder in spinal cord injury patients: Long-term functional results. Neurourol Urodyn. 2012; 31:672-676.
40. Studer UE, Burkhard FC, Schumacher M, et al. Twenty years experience with an ileal orthotopic low pressure bladder substitutelessons to be learned. J Urol. 2006; 176:161-166.

41. Webster GD, Maggio MI. The management of chronic interstitial cystitis by substitution cystoplasty. J Urol. 1989; 141:287-91.

42. Peeker R, Aldenborg F, Fall M. The treatment of interstitial cystitis with supratrigonal cystectomy and ileocystoplasty: difference in outcome between classic and nonulcer disease. J Urol. 1998; 159:1479-82.

43. Osman NI, Bratt DG, Downey AP, et al. A systematic review of surgical interventions for the treatment of bladder pain syndrome/interstitial cystitis. Eur Urol Focus. 2020; S24054569(20)30071-7.

44. Venn SN, Mundy AR. 'Nerve-sparing' cystectomy in women. Int Urogynecol J Pelvic Floor Dysfunct. 2000; 11:237-40.

45. Chiang PH, Huang YS, Wu WJ, Chiang CP. Orthotopic bladder substitution in women using the ileal neobladder. J Formos Med Assoc. 2000; 99:348-51.

46. Chong JT, Dolat MT, Klausner AP, et al. The role of cystectomy for non-malignant bladder conditions: a review. Can J Urol. 2014; 21:7433-41.

\section{Correspondence}

Chiara Borghi, MD (Corresponding Author)

borghi.chr@gmail.com

Margherita Manservigi, MD

mnsmgh@unife.it

Elena Sofia Milandri, MD

elenasofia.milandri@gmail.com

Pantaleo Greco, MD, Prof.

pantaleo.greco@unife.it

Department of Surgical Sciences, Section of Obstetrics and Gynecology,

Azienda Ospedaliero-Universitaria Sant' Anna, University of Ferrara,

via Aldo Moro 8, 44124 Cona (Ferrara), Italy

Carmelo Ippolito, MD

c.ippolito@ospfe.it

Department of Surgical Sciences, Section of Urology, Azienda

Ospedaliero-Universitaria Sant' Anna, University of Ferrara,

via Aldo Moro 8, 44124 Cona (Ferrara), Italy

Dell'Atti, $M D, P h D$

dellatti@hotmail.com

Division of Urology, Department of Clinical, Special and Dental Sciences,

University Hospital "Ospedali Riuniti" School of Medicine, Marche

Polytechnic University

via Conca 71, 60126 Ancona (Italy) 